Tobias J. Erb

\title{
8. Themenbereich synthetische Biologie: Top-down- und Bottom-up-Ansätze
}

Unter synthetischer Biologie versteht man Ansätze zur gezielten Erzeugung biologischer Systeme mit neuen Eigenschaften. Grundsätzlich wird zwischen zwei verschiedenen Ansätzen unterschieden. Im Top-down-Ansatz werden bereits natürlich vorkommende biologische Systeme (Zellbausteine, Zellen bzw. ganze Organismen) modifiziert, um diese neuen Eigenschaften zu erzeugen, während sich der Bottom-up-Ansatz mit dem Aufbau biomimetischer Systeme (künstliche Zellen, lebende Materialien) aus einzelnen (biologischen) Bausteinen beschäftigt. Dabei wird der Top-down-Ansatz sehr oft als eine logische Weiterführung klassischer molekularbiologischer bzw. gentechnologischer Methoden verstanden und gewinnt besonders im Rahmen der Bioökonomie immer mehr an Bedeutung. Im Gegensatz dazu ist die Bottom-up-synthetische-Biologie ein sehr junges Forschungsfeld an der Schnittstelle von Material- und Biowissenschaften, das noch im Entstehen ist. Die synthetische Biologie besitzt ein großes Anwendungspotenzial, das alle lebenswissenschaftlichen Bereiche, von der Medizin zur Landwirtschaft und Biotechnologie, umspannt. Im biotechnologischen Bereich findet sie bereits schon heute kommerzielle Anwendung.

\subsection{Die synthetische Biologie in einer sich wandelnden Bioökonomie}

Vor allem im Bereich der industriellen Biotechnologie gewinnen synthetisch-biologische Top-down-Ansätze gegenüber den klassischen Verfahren immer mehr an Bedeutung. In der klassischen Biotechnologie werden molekularbiologische bzw. gentechnische Ansätze gewählt, um mikrobielle Produktionsstämme zur Gewinnung verschiedener Zielmoleküle zu erzeugen. Diese Moleküle reichen von einfachen Ami- 
nosäuren bis hin zu komplexen Verbindungen, wie Polyketide oder Terpene, ${ }^{1}$ die beispielsweise als Pharmazeutika, Insektizide oder Geschmacks- und Geruchsstoffe eingesetzt werden. Um hohe Raum-Zeit-Ausbeuten zu erreichen, werden durch sogenanntes metabolisches (den Stoffwechsel betreffendes) Engineering natürlich existierende Stoffwechselwege in Mikroorganismen mittels molekulargenetischer Methoden gezielt verändert und der Stofffluss in Richtung Produkt umgelenkt. In den letzten Jahren wurden vermehrt auch ganze Gencluster bzw. Kombinationen mehrerer Gene unterschiedlichen biologischen Ursprungs in etablierte Wirtsstämme transplantiert, um chimäre oder artifizielle Biosynthesewege zu erzeugen, welche sogar die Synthese von Molekülen erlauben, die bisher nicht aus natürlichen Quellen gewonnen werden konnten (siehe unten).

Während in der Vergangenheit Mikroorganismen nach ihren natürlichen biosynthetischen Eigenschaften für die industrielle Produktion ausgewählt wurden, geht der Trend momentan hin zur Konzentration auf einige wenige, standardisierte Wirtsorganismen, denen gewünschte biosynthetische Eigenschaften neu implementiert werden. Solche standardisierten Produktionsstämme können immer einfacher erzeugt und charakterisiert werden (Eisenstein, 2016) aufgrund sinkender DNA-Synthese-Preise, der Möglichkeit, immer größere und komplexere DNA-Konstrukte zu assemblieren (Casini et al., 2015) - von Minichromosomen (Döhlemann et al., 2017; Carrillo et al., 2019) bis hin zu der Totalsynthese von bakteriellen Genomen (Hutchison et al., 2016; Robertson et al., 2021) -, sowie durch die Verfügbarkeit moderner genetischer Werkzeuge wie CRISPR/Cas (siehe hierzu Fehse, Kap. 6, und Fehse et al., Kap. 9) zur basengenauen bzw. randomisierten DNA-Modifikation in Kombination mit Automatisierungs- und Hochdurchsatz-Screening-Technologien. Dies ermöglicht es zum einen, die Entwicklungszyklen zu beschleunigen, zum anderen komplett neue, bisher nicht aus Mikroorganismen gewinnbare, Produkte zu erschließen.

Die Produktion des Anti-Malariamittels Artemisinin (bzw. der Vorläuferstufe Artemisinsäure) in Escherichia coli bzw. Bäckerhefe (Martin et al., 2003; Paddon et al., 2013) sowie die Herstellung von Opiaten und Tropan-Alkaloiden (natürlich vorkommenden organischen Verbindungen, die als Grundgerüst für verschiedene pharmakologische Wirkstoffe dienen), ebenfalls in Hefe (Galanie et al., 2015; Srinivasan/Smolke, 2020), sind Beispiele für die Gewinnung komplexer pharmazeutischer Verbindungen, die bisher mühsam aus Medizinpflanzen extrahiert werden mussten, aus einfachen Mikro-

1 Bei Polyketiden und Terpenen handelt es sich um Naturstoffe, eine heterogene Klasse von chemischen Verbindungen, die natürlicherweise in verschiedenen Organismen (u. a. Bakterien, Pilzen und Pflanzen) vorkommen und von denen viele aufgrund ihrer biologischen und pharmakologischen Eigenschaften für die im Text beschriebenen Anwendungen genutzt werden. 
organismen mittels synthetischer Biologie. Im Falle der Tropan-Alkaloide wurden in der Bäckerhefe insgesamt 34 Genommodifikationen vorgenommen (das Einbringen von 26 Genen aus der Pflanze in Hefe sowie das Ausschalten von weiteren 8 Hefe-Genen), was die Komplexität des gentechnischen Eingriffs zeigt (Srinivasan/Smolke, 2020).

Die Herstellung komplexer pharmazeutischer Verbindungen in Mikroorganismen ist nicht nur eine Vereinfachung gegenüber den bisherigen Extraktionsprozessen, sondern erlaubt auch eine gezielte Veränderung der Biosynthesewege. Dies eröffnet die Möglichkeit, die chemische Struktur der Produkte durch das Einbringen weiterer Enzyme zu modifizieren, um Verbindungen mit veränderter biologischer Aktivität zu erzeugen, was die Diversifizierung bzw. Entwicklung von maßgeschneiderten Molekülen mit neuen Eigenschaften (z. B. Medikamente, Antibiotika, Duftstoffe etc.) zukünftig deutlich effizienter machen wird (Courdavault et al., 2021).

Um diese synthetisch-biologischen Ansätze und deren zukünftigen Einsatz in der Biotechnologie weiter zu fördern, hat die US-amerikanische Regierung ein Forschungsprogramm aufgelegt, das es sich zum Ziel gesetzt hat, eine Vielzahl hochwertiger Moleküle, die entweder in der Herstellung sehr teuer bzw. aufwendig sind, nicht aus heimischen Quellen bezogen werden können oder durch traditionelle Synthesemethoden nicht zu erzeugen sind, mithilfe synthetisch-biologischer Ansätze herzustellen. Das Programm „Living Foundry“2 zielt auf die adaptierbare und skalierbare Bedarfsproduktion dieser Verbindungen durch „metabolisches Reprogrammieren“ biologischer Systeme. Dabei sollen im ersten Schritt neue Werkzeuge für die standardisierte Erzeugung verschiedener biologischer Plattformen entwickelt und diese dann anhand der Erzeugung von tausend unterschiedlichen Molekülen demonstriert werden. In ersten Versuchen wurde die grundsätzliche Eignung der Vorgehensweise anhand von $10 \mathrm{zu-}$ fällig ausgewählten Molekülen bereits gezeigt (Casini et al., 2018).

In Deutschland, das traditionell eine starke biotechnologische Industrie besitzt (ca. 650 Unternehmen mit mehr als 4 Milliarden Euro Umsatz) (Nuyken, 2021), gibt es bisher keine gezielten Programme, um eine nationale bzw. europäische Infrastruktur für vergleichbare synthetisch-biotechnologische Entwicklungs- bzw. Produktionsprozesse aufzubauen. Während vor allem angloamerikanische und asiatische Länder in die Etablierung von sogenannten „Biofoundries“ investiert haben, in denen die automatisierte Synthese und Charakterisierung biologischer Systeme erfolgt, gibt es auf deutscher bzw. kontinentaleuropäischer Ebene bisher keine vergleichbaren Initiativen. Lediglich das Novo Nordisk Foundation Center for Biosustainability ${ }^{3}$ an der Technical

2 Siehe unter: https://www.darpa.mil/program/living-foundries [09.06.2021].

3 Siehe unter: https://www.biosustain.dtu.dk/biofoundry [09.06.2021]. 
University of Denmark (DTU) und die MaxGENESYS Biofoundry ${ }^{4}$ am Max-Planck-Institut für terrestrische Mikrobiologie in Marburg sind erste Einrichtungen dieser Art.

\subsection{Biofoundries: Entwicklung neuer Technologien für eine neue Generation Bioingenieurinnen und -ingenieure}

Die DNA-Synthese ist eine Schlüsseltechnologie in der modernen Life-Science-Forschung. Viele Forschungsprojekte in der Bio(techno)logie und Medizin verlangen die Assemblierung und das Testen einzelner genetischer Elemente und genetischer Schaltkreise. Biofoundries sind Zentren, in denen DNA-Moleküle aus standardisierten genetischen Einzelteilen durch automatisierte und computergestützte Verfahren zusammengesetzt werden (Chao et al., 2017; Hillson et al., 2019; Holowko et al., 2021). Dies erlaubt es Wissenschaftlerinnen und Wissenschaftlern, mit geringem Aufwand tausende verschiedener genetischer Varianten zu erzeugen, die nachfolgend in Hochdurchsatzexperimenten weiter charakterisiert werden. Während traditionelles Klonieren und Transformieren bisher mit erheblichem Personal- und Zeitaufwand verbunden war, erleichtern Biofoundries die Erzeugung genetisch modifizierter Organismen um den Faktor 10-20 verglichen mit der händischen Laborarbeit (Hillson et al., 2019).

Die automatisierte Generierung und Hochdurchsatzcharakterisierung genetischer Elemente eröffnet nicht nur in der angewandten, sondern auch in der Grundlagenforschung neue Perspektiven (siehe auch Walter/Gasparoni, Kap. 3). Die Charakterisierung und Aufklärung genetischer und metabolischer Netzwerke durch das gezielte Neu-Assemblieren stellt eine Umkehr gegenüber dem vorherrschenden analytisch-destruktiven Ansatz in der Biologie dar, der bisher angewendet wurde, um die Funktionsweise biologischer Systeme und die Funktionen einzelner Gene zu studieren. Die hohe Zahl an Varianten, die mithilfe der DNA-Synthese und Automatisierung erzeugt werden können, erlaubt es, maschinelle Lernverfahren einzusetzen, um die Zusammenhänge und den Aufbau komplexer genetischer bzw. metabolischer Systeme zu verstehen bzw. zu optimieren. So können tausende Varianten eines biosynthetischen Stoffwechselwegs erzeugt werden, indem die einzelnen Gene, ihre Reihenfolge und ihr Expressionslevel systematisch variiert werden, um die optimale Kombination der einzelnen Elemente für die Produktion eines Zielmoleküls zu erzeugen. Ein weiteres Beispiel ist die Assemblierung komplexer genetischer Schaltkreise, um die Funktion genetischer Netzwerke zu verstehen und/oder neue Biosensoren zu erzeugen, die in der Diagnostik Anwendung finden könnten.

4 Siehe unter: https://www.mpi-marburg.mpg.de/927693/maxgenesys [09.06.2021]. 
Insgesamt kommt der automatisierten DNA-Synthese und -Charakterisierung eine wichtige Rolle zu, um die natürliche genetische Diversität bzw. Komplexität zu studieren und sie für eine industrielle Biotechnologie auszuschöpfen. Dabei muss die Charakterisierung genetischer Elemente nicht notwendigerweise im lebenden Organismus erfolgen. Inzwischen werden in den Biofoundries vermehrt In-vitro-TranskriptionsTranslations-Systeme bzw. zellfreie Extrakte eingesetzt, was die Erzeugung genetisch modifizierter Organismen und den damit verbundenen Ressourcen- und Zeitaufwand (vor allem in der Entwicklungs- und Charakterisierungsphase) weiter reduziert (Moore et al., 2017; Dudley et al., 2020).

In den letzten Jahren wurden mehrere Biofoundries an weltweit führenden Universitäten eingerichtet, unter anderem die MIT-Broad Foundry in den USA, die NUS Synthetic Biology Foundry in Singapur, die London Foundry am Imperial College (UK) und weitere dieser Zentren in USA, China und UK. Auch im industriellen Umfeld haben sich erste Anbieter etabliert, vor allem US-amerikanische Firmen, die die Entwicklung genetischer Systeme über Biofoundries als Servicedienstleistung anbieten und mit verschiedenen internationalen Firmen, darunter auch marktführenden Pharma- und Chemie-Konzernen aus Deutschland, kooperieren.

Dieser Trend zeigt, dass nach dem „Lesen der genetischen Information“ das „Schreiben genetischer Information" rasant an Bedeutung gewinnt, nicht nur in der Grundlagenforschung, sondern auch in der industriellen Anwendung. Im weiteren Verlauf der Entwicklung wird Digitalisierungs-, Automatisierungs- und Informationstechnologien zur Erzeugung und Analyse der biologischen Systeme eine Schlüsselrolle zukommen. Verglichen mit der Entwicklung anderer Hochtechnologien könnte dies mittelfristig zu einer Konsolidierung und Standardisierung der verwendeten Methoden, Organismen und genetischen Bauteile führen, was die Sicherheit und Innovationsgeschwindigkeit erhöhen könnte (Eisenstein, 2016; Sainz de Murieta et al., 2016). Der Gesetzgeber kann hier eine wichtige Rolle spielen, um den Übergang in eine vernetzte, digitalisierte Forschungs- und Produktionslandschaft in der Biotechnologie zu ermöglichen.

Dieser Übergang bedarf vor allem der Entwicklung und Genehmigung von Plattformorganismen (sogenannten biologischen "Chassis“), die einen sicheren Umgang, gute Umweltverträglichkeit, erleichterte Handhabung und vereinfachte Zulassung ermöglichen (de Lorenzo et al., 2021). Des Weiteren bedarf es der Charakterisierung und Festlegung genormter genetischer Bausteine, wie Promotern, Terminatoren, ribosoma- 
ler Bindestellen sowie Vektorsystemen ${ }^{5}$ (Canton et al., 2008; Silva-Rocha et al., 2013), die zum standardisierten Zusammenbau genetischer Konstrukte verwendet werden können. Das von der EU-Kommission geförderte Konsortium BioRoboost beschäftigt sich mit den wissenschaftlichen, technischen, ökonomischen, und gesellschaftlichen $\mathrm{He}-$ rausforderungen dieser Entwicklung. ${ }^{6}$ Ähnliche Projekte zur Standarisierung und deren Voraussetzungen bzw. Folgen wurden ${ }^{7}$ bzw. werden ${ }^{8}$ auch in den USA verfolgt. Eine große Herausforderung für die Standardisierung ist jedoch die Tatsache, dass das Verhalten biologischer Bausteine stark kontextabhängig ist (Cardinale/Arkin, 2012; Brophy/Voigt, 2014; Yeung et al., 2017) und komplexen Regeln unterliegt, die Gegenstand intensiver Untersuchungen sind. So bestimmt bspw. die DNA-Sequenz vor Promotern die Ableseaktivität (Carr et al., 2017) und die Position von Genen auf dem Chromosom die Expressionsrate (Scholz et al., 2019).

Einhergehend mit der technischen Weiterentwicklung wird eine neue Generation von synthetischen Biologinnen und Biologen bzw. Bioingenieurinnen und Bioingenieuren in Deutschland und Europa benötigt, um die Innovations- und Wettbewerbsfähigkeit der europäischen bzw. nationalen Bioökonomie zu erhalten. Neben der fachlichen Ausbildung an der Schnittstelle von Biologie, Ingenieurswissenschaften und Informationstechnologie sind dezidierte Schul- und Studienangebote zum Thema synthetische Biologie sowie das Vermitteln von Wissen an Bürgerinnen und Bürger ein wichtiges Ziel, um die noch junge Technologie bekannter zu machen und einen wissensbasierten, faktenorientierten Dialog mit Gesellschaft und Politik über die Risiken und Möglichkeiten der synthetischen Biologie als integraler Bestandteil einer zukünftigen Bioökonomie führen zu können (Mackelprang et al., 2021).

\subsection{Nachhaltige Synthese und Abfallströme als Rohstoffquelle mittels synthetischer Biologie}

Das Ziel der Bioökonomie ist es, eine biobasierte, am natürlichen Stoffkreislauf orientierte, nachhaltige Wirtschaftsform zu erreichen. Letzteres ist eine wichtige Voraussetzung, um die Nachhaltigkeitsziele der Vereinten Nationen bis zum Jahr 2030 erfül-

5 Bei Promotern handelt es sich um Bereiche auf der DNA, die das Ablesen und Umschreiben in RNA vermitteln; Terminatoren sind Bereiche, die es beenden. Ribosomale Bindestellen sind RNA-Bereiche, an denen die zelluläre Proteinbiosynthesemaschine, die Ribosomen, binden. Unter Vektorsystemen versteht man DNA-Ringe (Plasmide) zum gezielten Einbringen von Erbinformation in Zellen.

6 Siehe unter: https://cordis.europa.eu/project/id/820699 [09.06.2021].

7 Siehe unter: https://www.nist.gov/programs-projects/synthetic-biology-standards-consortium$\operatorname{sbsc}[09.06 .2021]$.

8 Siehe unter: https://www.nsf.gov/awardsearch/showAward?AWD_ID=1818248 [09.06.2021]. 
len zu können (BMBF, 2020; zur Nachhaltigkeit im Kontext der Grünen Gentechnologie siehe Renn, Kap. 23). Mittels synthetischer Biologie soll es unter anderem möglich werden, Produkte, die bisher nur über fossile Rohstoffe zugängig waren, über nachwachsende Rohstoffe zu gewinnen. Erste Erfolge konnten bereits demonstriert werden, wie z. B. die Biosynthese von 1,4-Butandiol, einem Lösungsmittel in dem Bakterium Escherichia coli (Yim et al., 2011), oder die Synthese des Virostatikums ${ }^{9}$ Didanosin durch einen neuartigen In-vitro-Stoffwechselweg (Birmingham et al., 2014). In beiden Fällen wurden zur Realisierung dieser „New-to-nature“-Stoffwechselwege Enzymaktivitäten benötigt, die bisher so nicht in der Natur vorhanden waren. Durch metabolische Retrosynthese und Protein-Engineering konnten diese nicht-natürlichen Aktivitäten in dem Gerüst bekannter Enzyme entwickelt werden. Durch die rasanten Fortschritte im Proteindesign (Siegel et al., 2010; Jeschek et al., 2016; Obexer et al., 2017; Vornholt et al., 2021) und der Entwicklung computerunterstützter Designverfahren (Carbonell et al., 2014) werden retro-(bio-)synthetische Ansätze zur Entwicklung maßgeschneiderter Biosynthesen zukünftig zunehmen (Erb et al., 2017).

Neben dem Erschließen neuer Produkte ist die Verwendung nachhaltiger Ausgangsmaterialien ein weiterer Fokus für die Entwicklung einer diversifizierten Bioökonomie. Während die meisten biotechnologischen Produktionsprozesse noch auf zuckerbasierten Fermentationen beruhen, wird weltweit eine Vielzahl von Ansätzen verfolgt, um neue Rohstoffquellen zu erschließen, $u$. a. verschiedene Abflussströme (z. B. Lignin, Cellulose, Plastik) und insbesondere das Treibhausgas $\mathrm{CO}_{2}$. Ein Beispiel für Letzteres ist die Verwertung von Synthesegas $\left(\mathrm{CO}_{2}, \mathrm{CO}, \mathrm{H}_{2}\right)$, das als Abfallprodukt in der Stahlproduktion entsteht bzw. aus regenerativen Energien mittels Elektrolyseuren ${ }^{10}$ erzeugt werden kann, durch anaerobe Mikroorganismen, wie Clostridien. ${ }^{11}$ Die Produktion der (Industrie-)Alkohole Ethanol, Butanol und Hexanol aus Synthesegas wurde bereits demonstriert (Haas et al., 2018; De Tissera et al., 2019) und die Verfahren werden zurzeit auf industrielle Größe skaliert. Durch synthetisch-biologische Ansätze wurde das Produktportfolio von Clostridien gezielt erweitert, sodass sie eine Palette weiterer Produkte, wie z. B. die Schlüsselchemikalie 3-Hydroxypropionsäure aus Synthesegas, herstellen können (Karim et al., 2020).

9 Ein Virostatikum hemmt die Verbreitung von Viren.

10 Als Elektrolyseur bezeichnet man eine Vorrichtung, mit der die Elektrolyse, also die Zerlegung von Wasser in seine Grundstoffe Wasserstoff und Sauerstoff, durchgeführt werden kann.

11 Clostridien bilden unter bestimmten Bedingungen (anaerob, also ohne Sauerstoff) Essigsäure (Acetat), Ethanol sowie andere höherkettige Säuren bzw. Alkohole aus Synthesegas. Diese Gasfermentation kann Säuren und Alkohole in jeder Region produzieren, ohne um Ressourcen für Nahrung oder Land zu konkurrieren (De Tissera et al., 2019). 
Im Rahmen der nationalen Wasserstoffstrategie könnten weitere hybride Verfahren entstehen, bei denen technisch erzeugter (,blauer“ bzw. „grüner“) Wasserstoff z. B. als Energiequelle von modifizierten Knallgasbakterien verwendet werden kann, um $\mathrm{CO}_{2}$ in Zielmoleküle, wie beispielsweise das Lösungsmittel Acetoin, umzuwandeln (Windhorst/Gescher, 2019). Alternativ könnte aus technischem Wasserstoff und $\mathrm{CO}_{2}$ erzeugtes Format (umgangssprachlich auch als Ameisensäure bezeichnet), Methanol (umgangssprachlich Holzalkohol) oder Methan als Energie- und Kohlenstoffquelle für Mikroorganismen dienen, die mittels synthetisch-biologischer Verfahren realisiert wurden. Kürzlich wurden Stämme von Escherichia coli bzw. der Industriehefe Pichia pastoris vorgestellt, denen synthetische Stoffwechselwege implementiert wurden, die es ihnen erlauben, direkt auf Format bzw. Methanol zu wachsen. Dadurch wird es möglich, diese bereits gut etablierten Plattformorganismen zukünftig für eine kohlenstoffneutrale oder sogar -positive Biotechnologie einzusetzen (Gassler et al., 2020; Kim et al., 2020). Weitere synthetisch-biologische Ansätze konzentrieren sich darauf, komplett neue $\mathrm{CO}_{2}$-bindende Enzyme und Stoffwechselwege zur effizienteren Umwandlung von $\mathrm{CO}_{2}$ zu generieren (Bar-Even et al., 2010; Schwander et al., 2016; Bernhardsgrutter et al., 2019; Scheffen et al., 2021). Diese Versuche zeigen konkrete Wege auf, über synthetische Biologie das Treibhausgas $\mathrm{CO}_{2}$ künftig als Rohstoffquelle zu gewinnen, Produktionsverfahren in der Syntheseindustrie klimafreundlicher zu gestalten und durch Sektorenkopplung eine (Teil-)Dekarbonisierung der Industrie zu erreichen.

\subsection{Bottom-up-Ansatz: Künstliche Zellen für neue Verfahren in der Biotechnologie und Medizin}

Alternativ zu den Top-down-Ansätzen, die sich auf das gezielte Umprogrammieren lebender Systeme fokussieren, widmet sich der Bottom-up-Ansatz der gezielten Synthese biologischer Systeme aus einzelnen Bausteinen. Das ultimative Ziel ist es dabei, die minimale Einheit des Lebens, die Zelle, aus unbelebten Einzelteilen zu rekonstruieren (Powell, 2018).

Dieser Ansatz wurde in den letzten Jahren in Deutschland durch ein durch das BMBF und die Max-Planck-Gesellschaft gefördertes Forschungsnetzwerk „MaxSynBio“12 vorangetrieben (Schwille et al., 2018). Andere europäische Länder, unter anderem die Niederlande, die Schweiz und das Vereinigte Königreich haben ähnliche Forschungsprogramme aufgelegt, was Europa zu einem Zentrum und Pionier der Bottom-up-synthetischen-Biologie hat werden lassen. In den USA wurde als Reaktion darauf erst kürz-

12 Siehe unter: https://www.maxsynbio.mpg.de/home [14.06.2021]. 
lich die „Build-a-synthetic-cell“-Initiative ins Leben gerufen, die sich ähnlichen Zielen wie die europäischen Initiativen widmet (Stano, 2018).

Im Mittelpunkt der Bottom-up-Ansätze steht zunächst die Erzeugung und Kontrolle einzelner „Module“ mit lebensähnlichen Eigenschaften und deren Kombination zu komplexeren Systemen, die sich in Raum und Zeit selbst organisieren. In einem inter- und transdisziplinären Ansatz werden Chemie, Biologie, Physik, Materialwissenschaften und Nanotechnologien zusammengeführt, um diese Module zu assemblieren. Hier kommt vor allem der Mikrofluidik eine wichtige Rolle zu, mit deren Hilfe zellgroße Tröpfchen bzw. Lipidhüllen erzeugt werden, die mit verschiedenen Eigenschaften ausgestattet werden können und als Vorläufer für eine „künstliche Zelle“ dienen (Weiss et al., 2018). Inzwischen konnten bereits Kompartimente mit metabolischer bzw. katalytischer (zum Vermitteln chemischer Reaktionen) Aktivität erzeugt werden (Beneyton et al., 2018), aber auch Protozellen, die zur lichtgetriebenen Kontraktion (sich mittels Licht zusammenziehen können) befähigt sind (Jahnke et al., 2020). Weitere Beispiele sind Protozellen mit Transkriptions-Translationssystemen zur Proteinproduktion (Noireaux et al., 2005) und die Entwicklung „künstlicher Chloroplasten“, die mithilfe von Licht $\mathrm{CO}_{2}$ umwandeln und als Alternative zur natürlichen Photosynthese dienen können (Lee et al., 2018; Miller et al., 2020).

Auch wenn es sich bei den ersten Studien zunächst lediglich um Grundlagenforschung und Machbarkeitskonzepte handelt, die sich mit der Erzeugung, Kontrolle und Analyse dieser „künstlichen Zellen“ beschäftigen, sind bereits mögliche zukünftige Anwendungen denkbar (Powell, 2018). Diese neuartigen Systeme könnten den Zugang zu neuen Informationsspeicherungs- bzw. -verarbeitungssystemen ermöglichen, die in der medizinischen Diagnostik ${ }^{13}$ oder Selbstoptimierung Anwendung finden (z. B. selbstständig mittels genetischer Schaltkreise das Vorhandensein von Krankheitsmarkern anzeigen oder neuartige Biokatalysatoren autonom entwickeln und verbessern). Weitere Einsatzmöglichkeiten sind räumlich und zeitlich organisierte multi-katalytische Systeme für die Biokatalyse bzw. chemische Synthese, sowie die Herstellung neuer Materialien, die mit der Umwelt interagieren und zur Selbstregeneration befähigt sind, für den technischen bzw. medizinischen Einsatz. In den nächsten ein bis zwei Dekaden wird die Weiterentwicklung der Bottom-up-synthetischen-Biologie aus der Grundlagenforschung heraus in erste Anwendungen erwartet.

13 Zur Gendiagnostik allgemein siehe Mundlos, Kap. 4. 


\subsection{Fazit}

Unter dem Sammelbegriff „synthetische Biologie“ werden verschiedene Ansätze zusammengefasst, die sich damit beschäftigen, neuartige biologische Systeme zu erzeugen. Molekularbiologische, bioinformatische und technologische Neuerungen lassen das Ziel, Organismen für verschiedenste Anwendungen maßgeschneidert zu erzeugen, immer näher rücken. Mit diesen neuen technologischen Möglichkeiten steht die Bioökonomie vor einem radikalen Wandel. Deutschland und Europa als bedeutender Pharma-, Chemie- und Biotechnologiestandort sollte in dieser Entwicklung aktiv tätig werden, um in einer der Schlüsseltechnologien zur Realisierung einer nachhaltigen Wirtschaftsform eine führende Rolle zu übernehmen.

\subsection{Literaturverzeichnis}

Bar-Even, A. et al. (2010): Design and analysis of synthetic carbon fixation pathways. In: Proc Nat Acad Sci USA 107(19): 8889-8894.

Beneyton, T. et al. (2018): Out-of-equilibrium microcompartments for the bottom-up integration of metabolic functions. In: Nat Commun 9: 2391.

Bernhardsgrutter, I. et al. (2019): Awakening the sleeping carboxylase function of enzymes: Engineering the natural $\mathrm{CO}_{2}$-binding potential of reductases. In: J Am Chem Soc 141(25): 9778-9782.

Birmingham, W. R. et al. (2014): Bioretrosynthetic construction of a didanosine biosynthetic pathway. In: Nat Chem Biol 10(5): 392-399.

$\mathrm{BMBF}=$ Bundesministerium für Bildung und Forschung (2020): Nationale Bioökonomiestrategie. Unter: https://www.bmbf.de/upload_filestore/pub/BMBF_Nationale_Biooekonomiestrategie_ Langfassung_deutsch.pdf [09.06.2021].

Brophy, J. A. N./Voigt, C. A. (2014): Principles of genetic circuit design. In: Nat Methods 11(5): 508-520.

Canton, B. et al. (2008): Refinement and standardization of synthetic biological parts and devices. In: Nat Biotechnol 26(7): 787-793.

Carbonell, P. et al. (2014). Retropath: automated pipeline for embedded metabolic circuits. In: Acs Synth Biol 3(8): 565-577.

Cardinale, S./Arkin, A. P. (2012): Contextualizing context for synthetic biology-identifying causes of failure of synthetic biological systems. In: Biotechnol J. 7(7): 856-866.

Carr, S. B. et al. (2017): Reducing DNA context dependence in bacterial promoters. In: PLOS ONE 12(4): e0176013.

Carrillo, M. et al. (2019): Design and control of extrachromosomal elements in Methylorubrum extorquens AM1. In: ACS Synth Biol 8(11): 2451-2456.

Casini, A. et al. (2015): Bricks and blueprints: methods and standards for DNA assembly. In: Nat Rev Mol Cell Biol 16(9): 568-576.

Casini, A. et al. (2018): A pressure test to make 10 molecules in 90 days: External evaluation of methods to engineer biology. In: J Am Chem Soc 140(12): 4302-4316.

Chao, R. et al. (2017): Engineering biological systems using automated biofoundries. In: Metab Eng 42: 98-108. 
Courdavault, V. et al. (2021): Metabolic engineering for plant natural products biosynthesis: new procedures, concrete achievements and remaining limits. In: Nat Prod Rep, Online-Publikation 10.05.2021. DOI: 10.1039/d0np00092b.

de Lorenzo, V. et al. (2021): For the sake of the bioeconomy: define what a synthetic biology chassis is! In: N Biotechnol 60: 44-51.

De Tissera, S. et al. (2019): Syngas biorefinery and syngas utilization. In: Adv Biochem Eng Biotechnol 166: $247-280$.

Döhlemann, J. et al. (2017): A family of single copy repABC-type shuttle vectors stably maintained in the alpha-proteobacterium Sinorhizobium meliloti. In: ACS Synth Biol 6(6): 968-984.

Dudley, Q. M. et al. (2020): In vitro prototyping of limonene biosynthesis using cell-free protein synthesis. In: Metab Eng 61: 251-260.

Eisenstein, M. (2016): Living factories of the future. In: Nature 531: 401-403.

Erb, T. J. et al. (2017): Synthetic metabolism: metabolic engineering meets enzyme design. In: Curr Opin Chem Biol 37: 56-62.

Galanie, S. et al. (2015): Complete biosynthesis of opioids in yeast. In: Science 349(6252): 1095-1100.

Gassler, T. et al. (2020): The industrial yeast Pichia pastoris is converted from a heterotroph into an autotroph capable of growth on $\mathrm{CO}_{2}$. In: Nat Biotechnol 38(2): 210-216.

Haas, T. et al. (2018): Technical photosynthesis involving $\mathrm{CO}_{2}$ electrolysis and fermentation. In: Nat Catalysis 1: 32-39.

Hillson, N. et al. (2019): Building a global alliance of biofoundries. In: Nat Commun 10(1): 2040.

Holowko, M. B. et al. (2021): Building a biofoundry. In: Synth Biol 6(1): ysaa026.

Hutchison, C. A., 3rd et al. (2016): Design and synthesis of a minimal bacterial genome. In: Science 351(6280): aad6253.

Jahnke, K. et al. (2020): Engineering light-responsive contractile actomyosin networks with DNA nanotechnology. In: Adv Biosyst 4(9): e2000102.

Jeschek, M. et al. (2016): Directed evolution of artificial metalloenzymes for in vivo metathesis. In: Nature 537(7622): 661-665.

Karim, A. S. et al. (2020): In vitro prototyping and rapid optimization of biosynthetic enzymes for cell design. In: Nat Chem Biol 16: 912-919.

Kim, S. et al. (2020): Growth of E. coli on formate and methanol via the reductive glycine pathway. In: Nat Chem Biol 16: 538-545.

Lee, K. Y. et al. (2018): Photosynthetic artificial organelles sustain and control ATP-dependent reactions in a protocellular system. In: Nat Biotechnol 36: 530-535.

Mackelprang, R. et al. (2021): Guiding ethical principles in engineering biology research. In: ACS Synth Biol 10(5): 907-910. 
Martin, V. J. et al. (2003): Engineering a mevalonate pathway in Escherichia coli for production of terpenoids. In: Nat Biotechnol 21: 796-802.

Miller, T. E. et al. (2020): Light-powered $\mathrm{CO}_{2}$ fixation in a chloroplast mimic with natural and synthetic parts. In: Science 368(6491): 649-654.

Moore, S. J. et al. (2017): Cell-free synthetic biology for in vitro prototype engineering. In: Biochem Soc Trans 45(3): 785-791.

Noireaux, V. et al. (2005): Toward an artificial cell based on gene expression in vesicles. In: Phys Biol 2(3): P1-8.

Nuyken, A. (2021): Biotech am Tipping Point. In welche Richtung entwickelt sich der Sektor nach der Pandemie? Biotechnologie-Report Deutschland 2021. Ernst \& Young GmbH, Eschborn/Frankfurt am Main.

Obexer, R. et al. (2017): Emergence of a catalytic tetrad during evolution of a highly active artificial aldolase. In: Nat Chem 9(1): 50-56.

Paddon, C. J. et al. (2013): High-level semi-synthetic production of the potent antimalarial artemisinin. In: Nature 496(7446): 528-532.

Powell, K. (2018): How biologists are creating life-like cells from scratch. In: Nature 563(7730): 172-175.

Robertson, W. E. et al. (2021): Creating custom synthetic genomes in Escherichia coli with REXER and GENESIS. In: Nat protocols 16: 2345-2380. DOI: 10.1038/s41596-020-00464-3.

Sainz de Murieta, I. et al. (2016): Toward the first data acquisition standard in synthetic biology. In: ACS Synth Biol 5(8): 817-826.

Scheffen, M. et al. (2021): A new-to-nature carboxylation module to improve natural and synthetic $\mathrm{CO}_{2}$ fixation. In: Nat Catalysis 4: 105-115. DOI: 10.1038/s41929-020-00557-y.

Scholz, S. A. et al. (2019): High-resolution mapping of the Escherichia coli chromosome reveals positions of high and low transcription. In: Cell Syst 8(3): 212-225.e9.

Schwander, T. et al. (2016): A synthetic pathway for the fixation of carbon dioxide in vitro. In: Science 354(6314): 900-904.

Schwille, P. et al. (2018): MaxSynBio: Avenues towards creating cells from the bottom up. In: Angew Chem 57(41): 13382-13392.

Siegel, J. B. et al. (2010): Computational design of an enzyme catalyst for a stereoselective bimolecular Diels-Alder reaction. In: Science 329(5989): 309-313.

Silva-Rocha, R. et al. (2013): The Standard European Vector Architecture (SEVA): a coherent platform for the analysis and deployment of complex prokaryotic phenotypes. In: Nucl Ac Res 41: D666D675. DOI: $10.1093 / \mathrm{nar} / \mathrm{gks} 1119$.

Srinivasan, P./Smolke, C. D. (2020): Biosynthesis of medicinal tropane alkaloids in yeast. In: Nature 585: 614-619.

Stano, P. (2018): Is research on „synthetic cells“ moving to the next level? In: Life 9(1): 3.

Vornholt, T. et al. (2021): Systematic engineering of artificial metalloenzymes for new-to-nature reactions. In: Sci Adv 7(4). DOI: 10.1126/sciadv.abe4208. 
Weiss, M. et al. (2018): Sequential bottom-up assembly of mechanically stabilized synthetic cells by microfluidics. In: Nat Mater 17(1): 89-96.

Windhorst, C./Gescher, J. (2019): Efficient biochemical production of acetoin from carbon dioxide using Cupriavidus necator H16. In: Biotechnol Biofuels 12: 163.

Yeung, E. et al. (2017): Biophysical constraints arising from compositional context in synthetic gene networks. In: Cell Syst 5(1): 11-24.e12.

Yim, H. et al. (2011): Metabolic engineering of Escherichia coli for direct production of 1,4-butanediol. In: Nat Chem Biol 7(7): 445-452. 